\title{
Efficient Algorithm to Enhance Lung Lobe Images using Fuzzy Filtering
}

\author{
Z.Faizal Khan \\ Research Scholar \\ Anna University of \\ Technology, Tirunelveli
}

\author{
S.N. Kumar \\ M.E. Applied Electronics \\ Anna University of \\ Technology, Tirunelveli
}

\author{
Dr.V.Kavitha \\ Director, University College \\ of Engineering, \\ Nagercoil Campus
}

\begin{abstract}
Preprocessing is done on the CT image of lung for removal of noise. A fuzzy filter is presented for the noise reduction of medical images corrupted with additive noise. The filter operation involves two stages. The first stage computes a fuzzy derivative for eight different directions. The second stage uses these fuzzy derivatives to perform smoothing with the fuzzification and De fuzzification operations along by weighting the contributions of neighboring pixel values. Both stages are based on fuzzy rules. The filter can be applied iteratively to effectively reduce heavy noise. In particular, the shape of the membership functions is adapted according to the remaining noise level after each iteration, making use of the distribution of the homogeneity in the image.
\end{abstract}

\section{INTRODUCTION}

Medical imaging is the technique that is used to create images of the human body (or parts and function thereof) for clinical purposes (medical procedures seeking to reveal, diagnose or examine disease) or medical science (including the study of normal anatomy and physiology)[3]. The CT images offer detailed information of lung cavities, which could be used for better surgical planning of treating Lung Cancer. Preprocessing is done to remove the noise from the isotropic CT image. The structure of the human lungs is shown here.

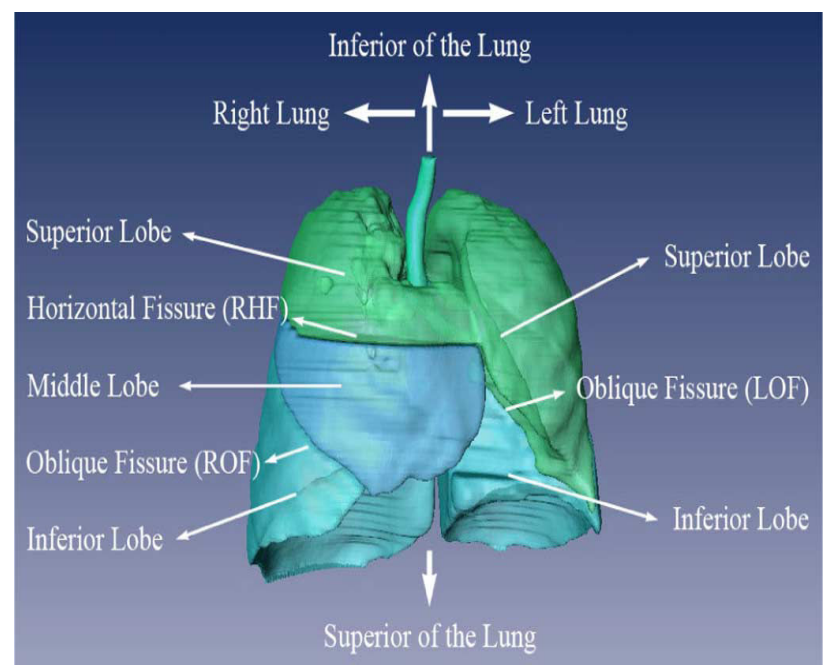

Fig 1: Structure of human lungs
Many existing methods are there for preprocessing. Filtering is the most fundamental operation in image processing and computer vision. The filtered image at a given location is a function of values of the input image in a small neighborhood of the same location. Assuming that images vary slowly over space, near pixels is likely to have similar values. But this assumption fails at regions that contain edges and image details (e.g. corners, lines, end of lines etc.) Most of the classical linear filters like the averaging low pass filters tend to blur and destroy the lines, edges and other fine image details

Wiener filter [2] is an optimization filter aimed to minimize the mean square error between the original image and the filtered counterpart. Since noise in CT images follows the Poisson distribution, which can be approximated using a Gaussian distribution for large number of occurrences. Fuzzy image processing [4] is the collection of all approaches that understand, represent and process the images, their segments and feature as fuzzy sets. The representation and processing depend on the selected fuzzy technique and on the problem to be solved. Fuzzy image processing involves three main stages. Image fuzzification, Membership modification, Image defuzzification. Fuzzy image processing using fuzzy techniques plays a very important role in image processing. Fuzzy techniques are important and powerful tools for knowledge representation and processing, and also managing the subjectivity and uncertainty very efficiently. The three important areas that are not perfect are Greyness ambiguity, Geometrical fuzziness, vague knowledge. Fuzzy Geometry, measures of Fuzziness and image information, fuzzy inference systems, fuzzy clustering, Fuzzy mathematical morphology, fuzzy measure theory, Fuzzy Grammars, neural fuzzy are some of important theoretical components of fuzzy image processing scheme.

Median filtering is similar to using an averaging filter, in that each output pixel is set to an average of the pixel values in the neighborhood of the corresponding input pixel. However, with median filtering, the value of an output pixel is determined by the median of the neighborhood pixels, rather than the mean. Median filtering is a specific case of order-statistic filtering, also known as rank filtering. The second order statistical filter can also be used. However the peak signal to noise ratio of these filters is low. In the proposed method, fuzzy filtering [1] can be used which is having high peak signal to noise ratio. A recursive fuzzy filtering technique [5] is presented to reduce additive Gaussian noise from color images using YIQ space. An adaptation scheme is developed to adapt the shape of 
membership functions of this filter according to remaining amount of noise after each iteration. Image de-noising is a technique [6] to reduce noises from a corrupted image. The aim of image denoising is to improve contrast of the image or perception of information in images for human viewers, or to provide better input for other automated image processing techniques.

A fuzzy filter [7] to remove impulse noise in an image with the combined ability of the RCM filter and fuzzy thresholding technique to preserve edges and fine details. The pixels lying outside the trimming range after ranking in the RCM filter are further tested for being noisy by the process of fuzzy thresholding. On CT, a fissure can have a variety of appearances [8], which makes its detection and segmentation challenging. Due to respiratory and cardiac motion, as well as partial volume effect, a fissure often appears as a ribbon-like structure several pixels wide, rather than the more typical configuration of a thin curvilinear opacity with sharp edges. For segmenting lung lobes depicted on 3-D CT examinations. The unique characteristic of this scheme is the representation of fissures in the form of implicit functions using Radial Basis Functions (RBFs)[9], capable of seamlessly interpolating "holes" in the detected fissures and smoothly extrapolating the fissure surfaces to the lung boundaries resulting in a "natural" segmentation of lung lobes. Takahiro [10] estimates the boundary surface between lung lobes by fitting curved surface. The fitting is performed with fuzzy control, and it searches the boundary where the density of tubular tissues is low. Image segmentation based contour models are also there in this paper fuzzy filter is used for the removal of noise from CT image of the lungs. The preprocessed image can be used for the lobe segmentation and for the extraction of the fissures.

The cad system for screening of lung cancer is developed for screening of lung cancer is developed to equal the ability of expert physicians [11][12].There are however no detection algorithms of the pulmonary lobes which are an important part in the detection of the pulmonary configuration in the clinical diagnosis. However fuzzy filter output can be processed further for lung lobe segmentation

by using suitable algorithm. Image segmentation based contour models are also there. Snake is an active contour model [13] which is used extensively in computer vision and image processing applications particularly to locate the object the boundaries. A pulmonary fissure is a boundary between the lobes in the lungs. Its segmentation is of clinical interest as it facilitates the assessment of lung disease on a lobar level. This paper [14] describes a new approach for segmenting the major fissures in both lungs on thin-section computed tomography (CT).An image transformation called "ridge map" is proposed for enhancing the appearance of fissures on CT. A curvegrowing process, modeled by a Bayesian network, is described that is influenced by both the features of the ridge map and prior knowledge of the shape of the fissure. Similar work has been done by Dimitri Van et al in [15].In addition i have enhanced the work for lung lobe images by by the process of fuzzification and defuzzification.Wiener filter is used for preprocessing for lung lobe segmentation; however the wiener filter is having low PSNR and high MSE while comparing with fuzzy filter

\section{FUZZY FILTERING}

The general idea behind the filter [15] is to average a pixel using other pixel values from its neighborhood, but simultaneously to take care of important image structures such as edges. The main concern of the proposed filter is to distinguish between local variations due to noise and due to image structure. In order to accomplish this, for each pixel we derive a value that expresses the degree in which the derivative in a certain direction is small. Such a value is derived for each direction corresponding to the neighboring pixels of the processed pixel by a fuzzy rule. If the fuzzy derivative is small, it is caused by the noise and if the fuzzy derivative is large, it is caused by the edge. Consequently, for each direction two fuzzy rules are applied (and thus distinguish between local variations due to noise and due to image structure), and that determine the contribution of the neighboring pixel values.

Consider the $3 \times 3$ neighborhood of a pixel as displayed in Fig. 2(a).

\begin{tabular}{|c|c|c|}
\hline $\mathrm{NW}$ & $\mathrm{N}$ & $\mathrm{NE}$ \\
\hline $\mathrm{W}$ & $(x, y)$ & $\mathrm{E}$ \\
\hline $\mathrm{SW}$ & $\mathrm{S}$ & $\mathrm{SE}$ \\
\hline
\end{tabular}

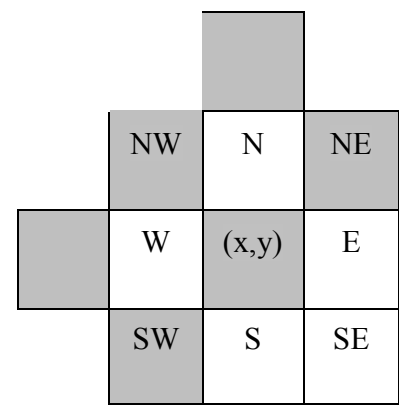

(a)

(b)

Fig. 2. (a) Neighborhood of a central pixel (x; y). (b) Pixel values indicated in gray are used to compute the "fuzzy derivative" of the central pixel $(\mathrm{x} ; \mathrm{y})$ for the NW-direction.

A simple derivative at the central pixel position $(\mathrm{x}, \mathrm{y})$ in the direction $\mathrm{D}(\mathrm{D} € \operatorname{dir}=\{\mathrm{NW}, \mathrm{W}, \mathrm{SW}, \mathrm{S}, \mathrm{SE}, \mathrm{E}, \mathrm{NE}, \mathrm{N}\}))$ is defined as the difference between the pixel at $(\mathrm{x}, \mathrm{y})$ and its neighbor in the direction $\mathrm{D}$. This derivative value is denoted by $(\mathrm{x}, \mathrm{y})$

For example

$$
\begin{aligned}
& \nabla_{N}(x, y)=I(x, y-1)-I(x, y) \\
& \nabla_{N W}(x, y)=I(x-1, y-1)-I(x, y)
\end{aligned}
$$

Next, the principle of the fuzzy derivative is based on the following observation. Consider an edge passing through the neighborhood of a pixel $(\mathrm{x}, \mathrm{y})$ in the SW-NE direction. The derivative value NW (x, y) will be large, but also derivative values of neighboring pixels perpendicular to the edge's direction can expect to be large.

For example, in the NW direction. We can calculate the values, $\nabla_{N W}^{F}(x, y)$ and $\nabla_{N W}^{F}(x+1, y-1)$ [see Fig. 2(b)]. The idea is to cancel out the effect of one derivative value which turns out to be high due to noise. Therefore, if two out of three derivative values are small, it is safe to assume that no edge is 
present in the considered direction. This observation will be taken into account when we formulate the fuzzy rule to calculate the fuzzy derivative values.

In Table 1, the overview of the pixels is used to calculate the fuzzy derivative for each direction. Each direction (column 1) corresponds to a fixed position (column 2); the sets in column 3 specify which pixels are considered with respect to the central $\operatorname{pixel}(\mathrm{x}, \mathrm{y})$.

Table1: Pixels to calculate the fuzzy in each direction

\begin{tabular}{|c|c|c|}
\hline Direction & Position & Set w.r.t $(\mathrm{x}, \mathrm{y})$ \\
\hline NW & $(\mathrm{x}-1, \mathrm{y}-1)$ & $\{(-1,1),(0,0),(1,-1)\}$ \\
\hline W & $(\mathrm{x}-1, \mathrm{y})$ & $\{(0,1),(0,0),(0,-1)\}$ \\
\hline SW & $(\mathrm{x}-1, \mathrm{y}+1)$ & $\{(1,1),(0,0),(-1,-1)\}$ \\
\hline S & $(\mathrm{x}, \mathrm{y}+1)$ & $\{(1,0),(0,0),(-1,0)\}$ \\
\hline SE & $(\mathrm{x}+1, \mathrm{y}+1)$ & $\{(1,-1),(0,0),(-1,1)\}$ \\
\hline E & $(\mathrm{x}+1, \mathrm{y})$ & $\{(0,-1),(0,0),(1,1)\}$ \\
\hline NE & $(\mathrm{x}+1, \mathrm{y}-1)$ & $\{(-1,-1),(0,0),(1,1)\}$ \\
\hline N & $(\mathrm{x}, \mathrm{y}-1)$ & $\{(-1,0),(0,0),(1,0)\}$ \\
\hline
\end{tabular}

To compute the value that expresses the degree to which the fuzzy derivative in a certain direction is small, the fuzzy set small is used. The membership function $m_{k}(u)$ for the property small is the following [see Fig. 2(a)]:

$M_{K}(u)= \begin{cases}1-\frac{|u|}{K}, & 0 \leq|u| \leq K \\ 0 & |u|>K\end{cases}$

For example, the value of the fuzzy derivative for thepixel in the NW direction is calculated by applying the following rule:

If $\left(\nabla_{N W}^{F}(x, y) \nabla_{N W}^{F}(x-1, y+1) \quad\right.$ is $\quad$ small and $\nabla_{N W}(x-1, y+1)$ is small $)$

$\mathrm{Or}$

$\left(\nabla_{N W}^{F}(x, y)\right.$ is small and $\nabla_{N W}^{F}(x+1, y-1)$ is small $)$

Or

$\nabla_{n w}(x-1, y=1)$ is small and $\nabla_{N W}(x+1, y-1)$ is

Small

then $\nabla_{N W}^{F}(x, y)$ is small

Eight such rules are applied to compute the degree of membership of the fuzzy derivatives $\nabla_{D}^{F}(x, y)$ to the represent the fuzzy set small. These rules are implemented in such a manner that minimum is used to represent the ANDoperator, and the maximum is used to represent the ORoperator. A defuzzification is not needed since the second stage, i.e., the fuzzy smoothing, directly uses the membership degrees to small.The robustness is achieved by the fuzzy derivative by combining multiple simple derivatives around the pixel and by making the parameter $\mathrm{K}$ adaptive.

\section{SMOOTHING BY FUZZIFICATION}

To compute the correction term $\Delta$ for the processed pixel value, a pair of fuzzy rules is used for each direction. The fuzzy rules are as follows: if no edge is assumed to be present in a certain direction, the (crisp) derivative value is used to compute the correction term.

The first part (edge assumption) can be realized by using the fuzzy derivative value, for the filtering it is needed to distinguish between positive and negative values.

For example, let us consider the direction NW, using the values $\nabla_{N W}^{F}(x, y)$ and $\nabla_{N W}^{F}(x, y)$.The two rules are fired and compute their truthness

$\lambda_{N W}^{+} \quad:$ if $\nabla_{N W}^{F}(x, y)$ is small and

$\nabla_{N W}^{F}(x, y)$ is positive then $\mathrm{c}$ is positive

$\lambda_{N W}^{-} \quad:$ if $\nabla_{N W}^{F}(x, y)$ is small and

$\nabla_{N W}^{F}(x, y)$ is negative then $\mathrm{c}$ is negative

The correction term is given as follows where D€dir (for all directions)

$$
\Delta=\frac{L}{8} \sum_{D \in d i r}\left(\lambda_{D}^{+}-\lambda_{D}^{-}\right)
$$

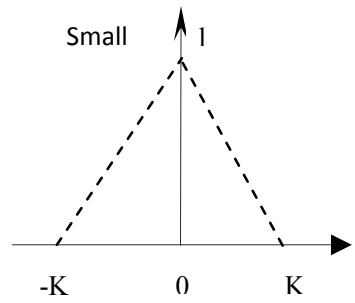

(a)

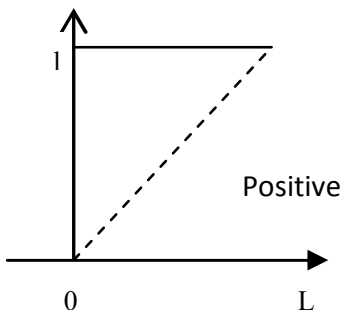

(b)

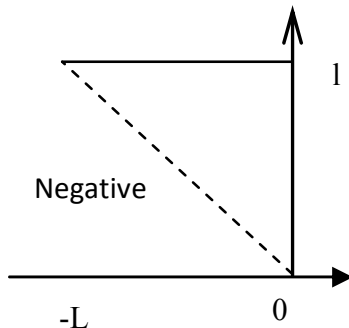

(c) 
Fig.3. Membership functions (a) small, (b) positive, and (c) negative.

\subsection{Fuzzification and Defuzzification scheme}

The fuzzification and defuzzification steps are due to the lack of possessing fuzzy hardware.So that the coding and decoding (Fuzification and defuzzification )of the results makes use of various fuzzy techniques. These fuzzy techiniques are used to obtain the membership function of the images. After the transform of image datas from gray-level plane to the membership plane, the appropriate fuzzy techniques modify the membership values (fuzzification).Fuzzification can be computed using the following equation.

$$
\forall x k: \mu_{L X} *\left(x *_{k}\right)=\left\{\begin{array}{l}
1 ; \text { if } x=x_{k^{*}} \\
0 ; \text { otherwise }
\end{array}\right.
$$

The value of LX can br determined by identifying the corresponding neighboring pixels as

$$
L X_{k}^{*}=\int_{x} \mu_{L X} *\left(x_{k} *\right) / x
$$

For each block (B), compute the standard deviation using the following equation:

$$
\sigma_{i}=\sqrt{\left.\frac{1}{r \times r} \sum_{\left(x^{\prime} y^{\prime}\right) \in B_{i}} I\left(x^{\prime}, y^{\prime}\right)-m i\right)^{2}}
$$

Where $m_{i}$ is the mean value of block $\left(B_{i}\right), I\left(x^{\prime}, y^{\prime}\right)$ is the pixel data inside the block $\left(\mathrm{B}_{\mathrm{i}}\right)$ Then, find omin which represents the minimum $(\sigma)$ in all blocks.

After the membership function was obtained defuzzify it with the defuzzification operator.Defuzzification can be obtained by the following equation

$$
y^{*}=\frac{\int_{y} y \cdot \mu_{L X}(y) d y}{\int_{y} \mu_{L X}(y) d y}
$$

This scheme can be applied before each iteration to obtain the adaptive parameter $(\mathrm{q})$, which determines the shape of the membership function small.

\section{RESULTS}

The efficency of the fuzzy filter along with the fuzzification and defuzzification operators is determined from the PSNR value. This ratio is often used as a quality measurement between the original and preprocessed image. The higher the PSNR, the better the quality of the preprocessed image.The Mean Square Error (MSE) and the Peak Signal to Noise Ratio (PSNR) are the two error metrics used to compare image quality.The MSE represents the cumulative squared error between the compressed and the original image, whereas PSNR represents a measure of the peak error. The lower the value of MSE, the lower the error.

$M S E=\frac{\sum_{M, N}\left[I_{1}(m, n)-I_{2}(m, n)\right]^{2}}{M^{*} N}$
$\mathrm{M}$ and $\mathrm{N}$ are the number of rows and columns in the input images, respectively. Then the block computes the PSNR using the following equation:

$$
\mathrm{PSNR}=10 \mathrm{LOG}_{10}\left(\mathrm{R}^{2} / \mathrm{MSE}\right)
$$

$\mathrm{R}$ is the maximum fluctuation in the input image data type. For example, if the input image has a double precision floating-point data type, then $R$ is 1 . If it has an 8 -bit unsigned integer data type, $\mathrm{R}$ is 255 .

Table 2:Characterstics of different types of filters

\begin{tabular}{|l|l|l|}
\hline Type of the filter & PSNR & MSE \\
\hline 2D Order Statistics Filter & 16.6 & 1422 \\
\hline Median Filter & 20 & 726.6 \\
\hline Wiener Filter & & \\
\hline Fuzzy Filter & 31.894 & 41.990 \\
\hline
\end{tabular}

The PSNR and MSE for different types of filters are as follows. The input CT image of the lungs and the histogram of the image is shown in Fig4.

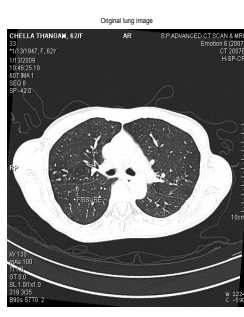

(a)

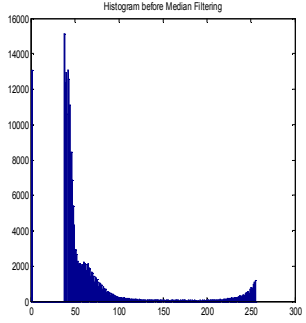

(b)
Fig4.(a)input CT image of the lngs,3.(b)Histogram of the input $\mathrm{CT}$ image

The preprocessed image by 2D order statistics filtering and its histogram are shown below. ThePSNR value of statistics filter is 16.6 .

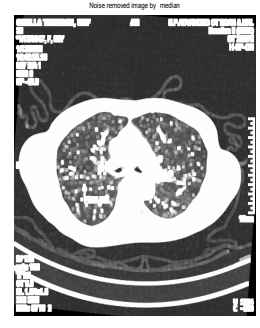

(a)

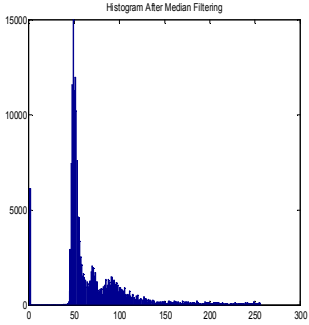

(b)
Fig5.(a)Preprocessed image by 2D order statistics filtering,(b)Histogram after statistics filtering 
The preprocessed image by median filtering and its histogram are shown below.The PSNR value of statistics filter is 20 .

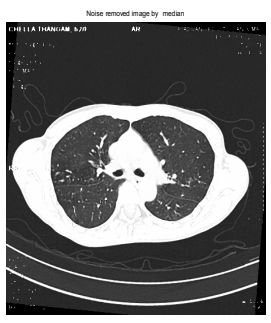

(a)

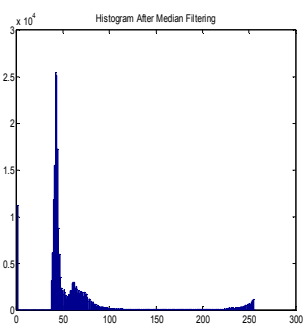

(b)
Fig6.(a)Preprocessed image by median filtering (b)Histogram after median filtering

The preprocessed image by wiener filtering and its histogram are shown below. The PSNR value of wiener filter is 31.894 .

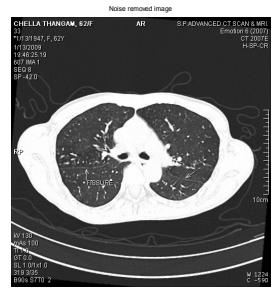

(a)

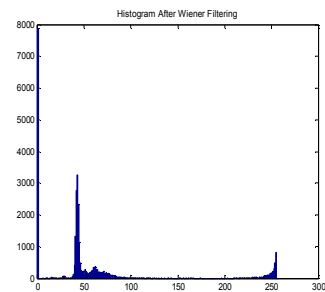

(b)
Fig7.(a)Preprocessed image by wiener filtering,(b) Histogram after wiener filtering

The preprocessed image by fuzzy filtering and its histogram are shown below. The PSNR value of fuzzy filter is 34.57 .

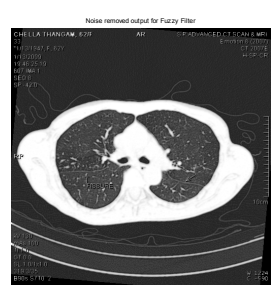

(a)

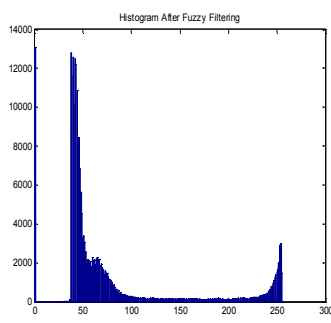

(b)
Fig8.(a)Preprocessed image by fuzzy filtering, (b) Histogram after fuzzy filtering

The bar graph of PSNR value of different types of filters are shown here. The fuzzy filter is having highest PSNR value.

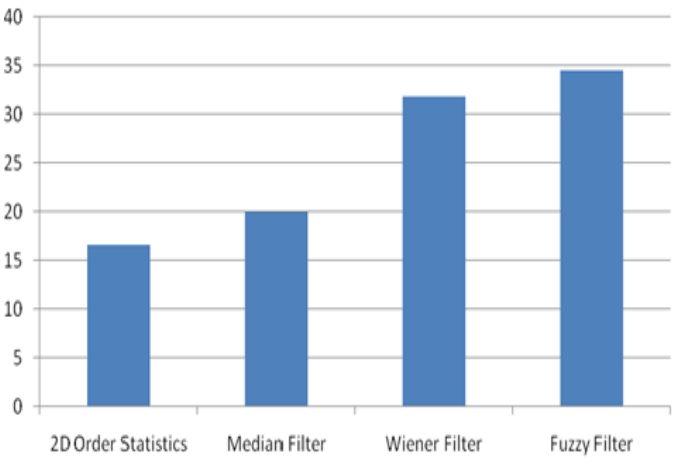

Fig 9: Bar Graph of PSNR value for different types of filter

The bar graph of MSE value of different types of filters are shown here. The fuzzy filter is having lowest MSE value.

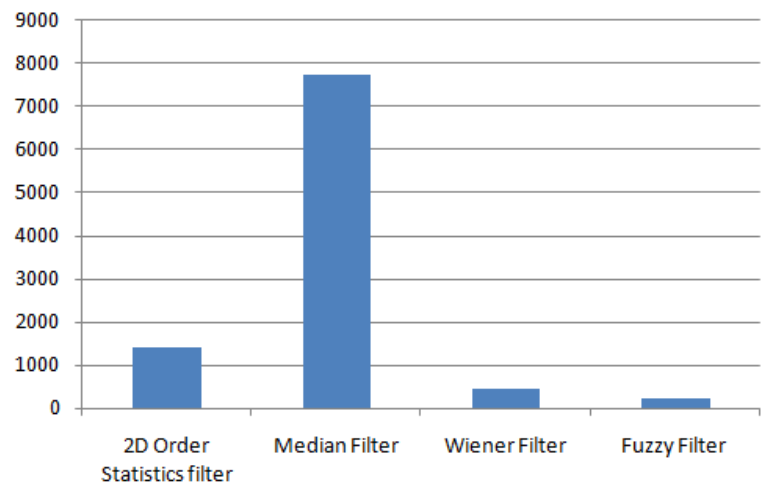

Fig 10: Bar Graph of MSE value for different types of filter

Thus the fuzzy filter is having high PSNR and low MSE while compared to that of other filters

\section{CONCLUSION}

The fuzzy filter can be used for preprocessing the CT image of lungs.The preprocessed $\mathrm{CT}$ image is subjected to sutiable algorithm for lung lobe segmentation and fissure extraction which provides a preplanning for surgery. While performing the filtering originality of the image have to be. preserved. The fuzzy filtering performs the noise removal by preserving the edges.The fuzzyfilter is having a PSNR of 34.57 and MSE of 22.63 for the typical lung CT image, which indicates that it produce better results than other filters.

\section{REFERENCES}

[1] Dmitri Van De Ville, Mike Nachtegael DietrichVan der Weken, Etienne E Kerre Kerre,Wilfried Philips and Ignace Lemahieu,"Noise Reduction by Fuzzy Image Filtering", IEEE transactions on fuzzy systems,vol. 11,no.4, August 2003

[2] Qiao wei,Yapoing Hu,'Segmentation of Lung Lobes in High Resolution Isotropic CT images",IEEE transactions on biomedical engineering,Vol56,No5,May 2009.

[3] B.N.Raasch, E.W. Carsky, E. J. Lane, J. P.O'Callaghan, and E. R. Heitzman, "Radiographic anatomy of the 
interlobar fissures: A study of 100 specimens," Amer. J. Roentgenol., vol. 138, pp. 647-554, 1982.

[4] Jiantao Pu, Bin Zheng, Joseph K.Leader,Carl Fuhrman, Friedrich Knollmann, Amy Klym, and David Gur, "Pulmonary Lobe Segmentation in CT Examinations Using Implicit Surface Fitting", IEEE transactions on medical imaging, vol. 28, no. 12, December 2009

[5] Takahiro Ohkawal, Syoji Kobashi', Katsuya Kondo',Yutaka Hatal, Tonioham Nakaiio "Tubular Tissue-Based Segmentation Of Lung Lobes From Chest Mdct Images", Cancer and horacicSurgery, Graduate School of Medicine, Okayama University, 2003.

[6] M. Kubo, N. Niki, S. Nakagawa, K. Eguchi, M Kaneko, N. Moriyama,H. Omatsu, R. Kakinuma and N. Yamaguchi, "Extraction algorithm of pulmonary fissures from thin-section CT images based on linear feature detector method," IEEE Trans. Nucl. Sci., vol. 46, no. 6, pp. 2128-2133, Dec. 1999.

[7] M. Kubo, Y. Kawata, N. Niki, K. Eguchi, H.Ohmatsu, R. Kakinuma, M. Kaneko, M. Kusumoto, $\mathrm{N}$. Moriyama, K. Mori, and H. Nishiyama, "Automatic extraction of pulmonary fissures from multidetector-row CT images," in Proc. IEEE Int Conf. Imag. Process. (ICIP), pp. 1091- 1094, 200.

[8] Yoshinori itai,seji ishikawa,"Automatic segmentation of lung areas based on snakes and extraction of abnormal areas", 2005

[9] J.Wang, M. Betke, and J. P. Ko, "Pulmonary fissure segmentation on CT,"Med. Imag. Anal,vol. 10, pp. 530 547, 2006.
[10] Segmentation of Lung Lobes in Isotropic CT Images Using Wavelet Transformation Q. Wei, Student Member, IEEE, Y. Hu, Member, IEEE G. Gelfand, and J. H. Mac Gregor,Proceedingsof the 29th Annual InternationalConference of the IEEE EMBSCité Iternationale, Lyon,France August 23-26, 2007.

[11] L.Zhang, E. A. Hoffman, and J. M. Reinhardt, "Atlasdriven lung lobe segmentation in volumetric X-ray CT images," IEEE Trans. Med. Imag., vol. 25, no. 1, pp. 1-16, Jan. 2006

[12] M.Hari Krishnan, R. Viswanathan, "Applications of Advanced Fuzzy Logic Techniques in Fuzzy Image Processing Scheme", Advances in Fuzzy Mathematics, Volume 5, Number 1 (2010), pp. 71-76, Research India Publications

[13] Munther N. Baker, Ali A. Al-Zuky ," Color Image Noise Reduction Using Fuzzy Filtering “,Journal of Engineering and Development, Vol. 12, No. 2, June (2008)

[14] Ayyaz Hussain, M. Arfan Jaffar, Zia Ul- Qayyum, Directional Weighted Median Based Fuzzy Filter For Random-Valued Impulse Noise Removal", Icic International C2010 Issn 2185-2766, Volume 1, Number 1, September 2010

[15] Dimitri Van De Ville, Member, IEEE, Mike Nachtegael, Dietrich Van der Weken, Etienne E.Kerre, Wilfried Philips, "Noise Reduction by Fuzzy Image Filtering", IEEE transactions on fuzzy systems, vol. 11, no. 4, august 2003 